\title{
FLEXIBLE FREE STANDING SU-8 MICROFLUIDIC IMPEDANCE SPECTROSCOPY SENSOR FOR 3D MOLDED INTERCONNECT DEVICES APPLICATION
}

\author{
M.-P. Schmidt ${ }^{1}$, A. Oseev ${ }^{1}$, C. Engel ${ }^{2}$, A. Brose ${ }^{1}$ and S. Hirsch ${ }^{3}$ \\ ${ }^{1}$ Institute of Micro and Sensor Systems/ Otto von Guericke University Magdeburg, Universitätsplatz 2, \\ 39106 Magdeburg, Germany, \\ marc-peter.schmidt@ovgu.de \\ ${ }^{2}$ TEPROSA GmbH, Mittagstr.16p, 39124 Magdeburg, Germany, \\ ${ }^{3}$ Department of Engineering/ University of Applied Sciences Brandenburg, Brandenburg, Germany,
}

\begin{abstract}
:
Current contribution reports a new microfluidic impedance spectroscopy sensor that is applicable for direct attachment on 3D molded interconnect devices (3D-MID). The Sensor is made of flexible SU-8 polymer featured by the system of electrodes structured directly on SU-8. Sensor chip is able to be directly soldered on MID due to electroless plated Nickel contact pads. Due to opposite electrodes design and one side direct shielded attachment, sensor is able to be applied for measurements in intensive electromagnetic environment. To the best of our knowledge none of such sensors has been previously reported.
\end{abstract}

Key words: SU-8, 3D-MID, microfluidic impedance spectroscopy

\section{Introduction}

Impedance spectroscopy is a well-known method for liquid analysis [1]. For decades it is applied in microfluidic systems for liquid properties determination [2,3]. Depending on the microchannel material, microfluidic sensors can be performed on a wafer basis $[4,5]$ or it can be completed as a free-standing structure that is applicable for 3D attachment. When microfluidic sensor is completed on a wafer level, it demonstrates a number of advantages, but at the same time remains not applicable for usage within 3D systems.

During the last years, molded interconnection devices (MIDs) are attracting considerable attention. Structures completed within MID technology can be applied as a 3D detail of a system, but at the same time having all required electrical interconnections, circuit elements and sensors directly on it. Due to the injection molding and metallization process a wide range of 3D-shaped substrates are fabricable, Fig. 1. In order to be applied in such 3D systems, it is required from sensor to have a flexible origin with further possibility for 3D direct MID attachment.

Manufacturing of free-standing microfluidic structures can be completed under different approaches [6]. PDMS material is well known regarding microfluidic structures manufacturing [7]. It is considerably low cost material, but it cannot be directly structured with photolithography. In order to structure PDMS, different techniques such as micro-molding are needed to be applied that can considerably affect resolution of a final microfluidic structures.

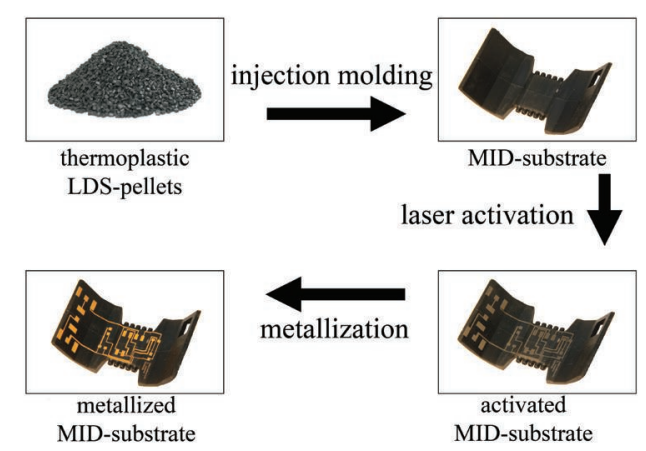

Fig. 1. Fabrication process of a microfluidic 3D-MID with laser direct structuring LDS

Polyimide is also widely applied as a material for microfluidic sensors especially for medical applications [8]. The same way as PDMS, polyimide does not provide a possibility for direct structuring with lithographical methods and normally, dry etching process is needed to be performed for that. 
$\mathrm{SU}-8$ is already very well-known photoresist that is widely used as a construction material for microfluidic structures, sensors and wide variety of different applications. Based on EPON-Resin SU-8 is chemically stable and optically transparent material with controllable mechanical properties through its processing and possibility to be directly structured with a standard photolithography process $[9,10]$.

Lots of wafer based SU-8 microfluidic structures have been already published [11,12], but completely free-standing SU-8 microfluidic structures are rarely declared due to certain complexity of structuring of metal layers on SU8 and releasing step technology for millimeter scale structures.

In [13] the fabrication of a SU-8 thermal flow sensor with manual Kapton film releasing was demonstrated. Releasing was completed only from one side of the channel finalizing pure SU8 encapsulation of microfluidic structures. Completed microfluidic sensor, after releasing step, is still remains on second PMMA holding wafer and is not applicable for 3D attachment. The same Kapton film releasing steps were applied in [14] where final SU-8 free-standing cantilever structures were embedded in a SU-8 on silicon based structures and in [15] where SU-8 multilayer microstructures were adhesive bonded and released with Kapton film. In all of referred contributions, final microfluidic structures remained on a handling wafer that makes them not applicable for 3D attachment.

Recently, the new nanoscale sacrificial releasing layer Omnicoat ${ }^{\mathrm{TM}}$ was introduced and applied for SU-8 structures releasing [16]. Initially aimed to be used for stripping of SU-8 molds in which metal structures are electroplated, it has become a very convenient and considerably clean method for SU-8 microfluidic structures wet releasing [17]. In current contribution we demonstrate SU-8 based technology with Omnicoat ${ }^{\mathrm{TM}}$ releasing step that affords to achieve completely freestanding SU-8 based microfluidic sensors.

In comparison to previously published results, in current contribution we demonstrate an impedance spectroscopy sensor with metal electrode structures completely integrated in SU-8 and released from both sides resulting completely free-standing and flexible SU-8 based sensor. Manufactured SU-8 free standing microfluidic sensor is applicable for subsequent 3D-MID attachment that gives an opportunity to create reduced-scale sensor devices for 3D applications.

\section{Fabrication of the sensor device}

The fabrication process of the sensor was divided into three parts, Fig. 2. First the fabrication was completed with silicon wafer where SU-8 microfluidic structures and sensor bottom electrodes were defined Fig.2 Part 1. It starts with the cleaning of the $100 \mathrm{~mm}$ silicon wafer with an HF $1 \%$ dip for $1 \mathrm{~min}$. An adhesion layer of Omnicoat ${ }^{\mathrm{TM}}$ was spun on the clean substrate and baked for $1 \mathrm{~min}$ on a hotplate at $200{ }^{\circ} \mathrm{C}$. In order to improve releasing speed, thickness of Omnicoat ${ }^{\mathrm{TM}}$ was enlarged by subsequent multilayer coating. Next SU-8-50 handling layer was coated on the Si wafer in a thickness of $50 \mu \mathrm{m}$ without any structures. After the first SU-8 layer coating the bottom electrode (Titanium $(30 \mathrm{~nm})$ and Platinum $(300 \mathrm{~nm}))$ of the sensor chip was deposited on the polymer and structured via Lift-Off. The surface of the SU-8 was pretreated with plasma of $\mathrm{O}_{2} / \mathrm{SF}_{6}$ to improve the adhesion between the polymer and metal electrodes. After stripping the photoresist layer a $50 \mu \mathrm{m}$ thick SU-8-50 layer was spin coated and structured to implement the microfluidic channels. To avoid thermal mechanical stress all baking processes were temperature ramped.
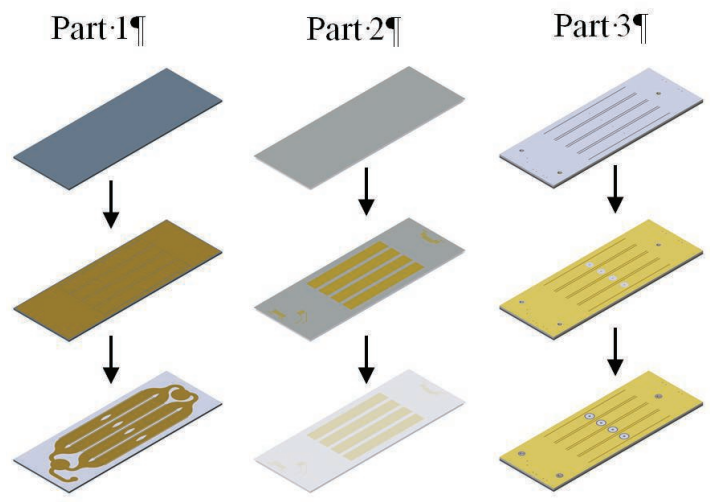

Fig. 2. Fabrication of the complete sensor, Part 1 fabrication of the bottom electrode and the microfluidic channels of the sensor chip, Part 2 manufacturing of the top electrode and the SU-8 top cap, Part3 establishing of the bonding, dry etching and plating of the contact structures

Next part of sensor manufacturing was including fabrication of sensor top electrodes structures on SU-8/glass wafer Fig.2 Part 2. Borosilicate glass was cleaned in a solution of $\mathrm{H}_{2} \mathrm{SO}_{4}$ and $\mathrm{H}_{2} \mathrm{O}_{2}$ at $135{ }^{\circ} \mathrm{C}$ for 10 min and then coated with Omnicoat ${ }^{\mathrm{TM}}$ for further releasing of the next $50 \mu \mathrm{m}$ thick layer of SU-8-50. A plasma treatment was performed to activate the surface of the polymer layer improving the adhesion of subsequently sputtered $\mathrm{Ti} / \mathrm{Pt}$ metal layer that was further Lift-Off structured. The layer was then covered with a thin film of SU-8-5. A second exposure step (dose of $250 \mathrm{~mJ} / \mathrm{cm}^{2}$ ) 
opened the possibility for an additional crosslinking of the SU-8-5 layer in the following bonding step. Processed within first and second steps wafer based structures were adhesive bonded after the exposure with an assistance of a SUSS MA6/BA6 mask aligner and a SUSS SB6e substrate bonder. After the bonding step the glass wafer was released in an etchant.

On top of the SU-8 a $200 \mathrm{~nm}$ Aluminum layer was sputtered and structured via lithography and etching. The Al mask was used in Oxford Instruments PlasmaSystem100 dry etching process to open the polymer layers and establish the entry for the top sensor electrode contact and the sidewalls for the shielding. To avoid any thermal mechanical stress of the SU8 layer during the plasma etching process the Si handling wafer was cooled with helium and the etching was divided into steps of $30 \mathrm{sec}$ with the mixture of $\mathrm{SF}_{6} / \mathrm{O}_{2}$. The mask material was stripped and a new metal layer of $30 \mathrm{~nm} \mathrm{Pt}$ and $500 \mathrm{~nm} \mathrm{Al}$ were structured with a Lift-Off process to connect the top electrodes and establish the 3D-shielding. To realize the solder connections a final photoresist Ti Spray was spray coated with a SUSS Delta Altaspray. By using an electroless metallization bath the final layers of nickel and gold were plated. After the metallization the completed SU-8 multilayer structures were separated from the Si handling wafer by a releasing step of the Omnicoat ${ }^{\mathrm{TM}}$ in MF-319 developer.

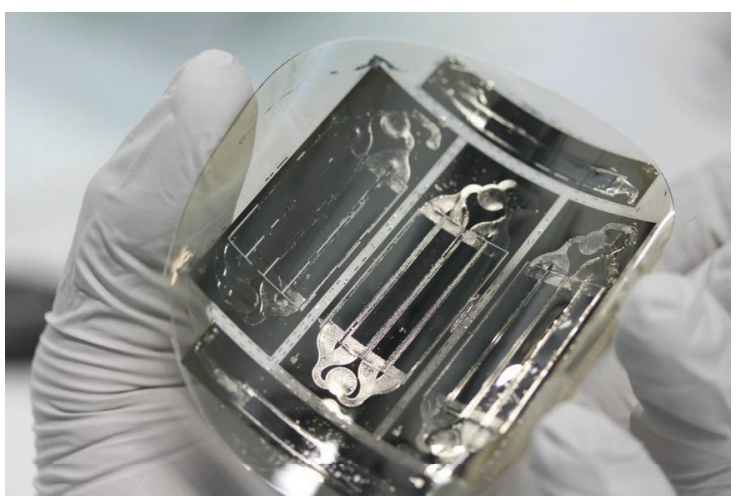

Fig. 3. Completed SU-8 based free standing impedance spectroscopy sensors on a $100 \mathrm{~mm}$ substrate with different designs.

\section{Experimental results}

Completely fabricated free standing microfluidic sensors intended for further dicing are shown in Fig. 3. Sensors are completed within a 4" size and demonstrate sufficient flexibility for 3D attachment. SEM image of a section of free standing microfluidic channel is shown in Fig. 4. As it can be seen, there is no boundaries between different layers of SU-8 can be observed and side walls are almost vertical.
Bonding area is completely closed without defects even after sample dicing.

The self-developed 3D-MID platform was injection molded in our lab under help of an ARBURG 320s equipment. The 3D-MID substrate integrates all electrical as well as fluidic interconnections between microfluidic chip, measuring device and sensor itself and is shown in Fig. 5.

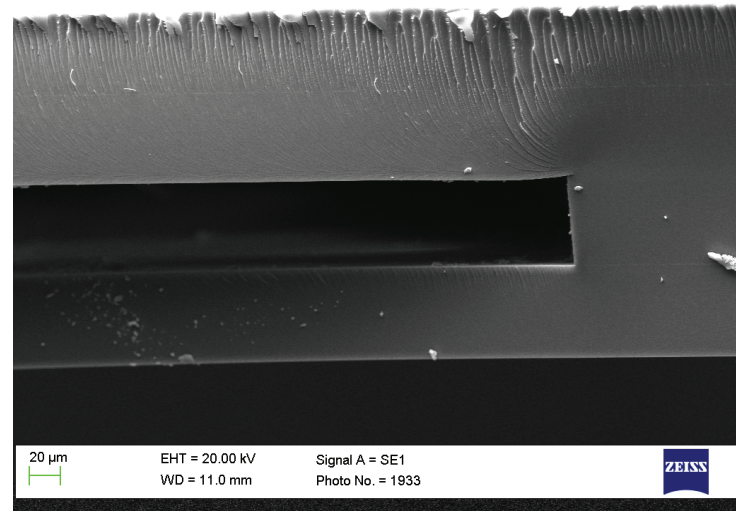

Fig. 4. SEM image of a free standing microfluidic channel.

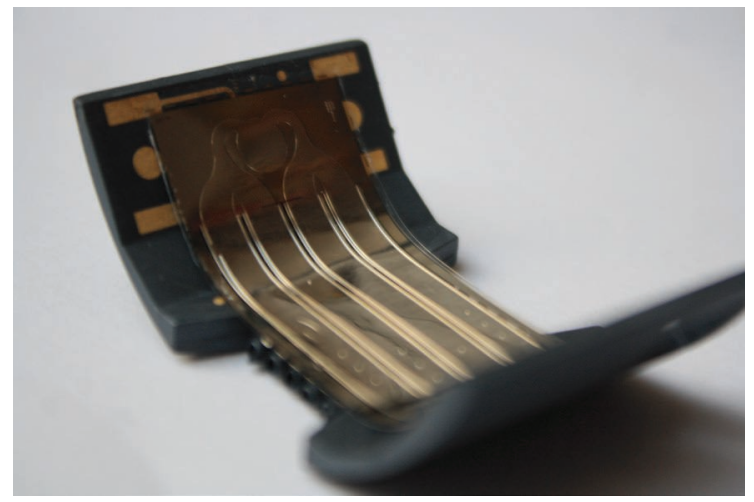

Fig. 5. Impedance spectroscopy sensor directly attached on a 3D-MID.

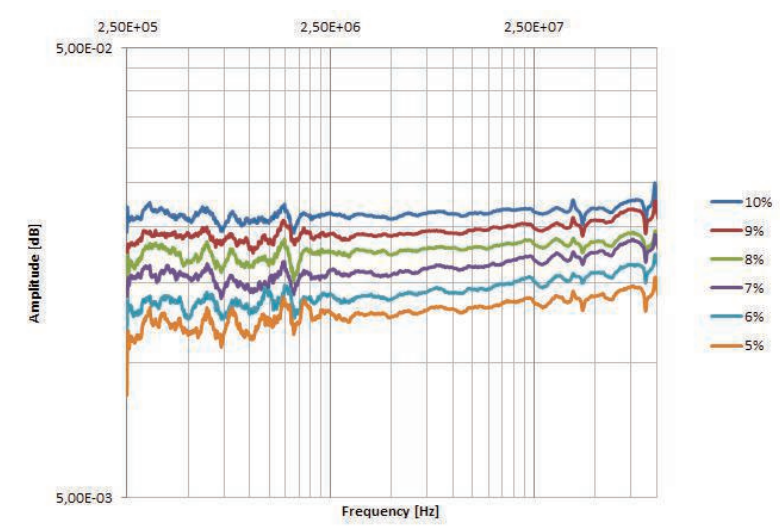

Fig. 6. Sensor $S_{21}$ parameter measurement results of mixture of water and ethanol in a frequency range of $0,25 \mathrm{MHz}$ and $250 \mathrm{MHz}$.

Due to sensor's plated contact pads, it is able be directly attached to 3D-MID platform by solder process without additional chip wire 
bonding. The microfluidic part of the 3D-MID was connected to a fluid system which contained a micro pump, a reservoir and a temperature control unit. The electrical ports of the MID were wired with a FPGA based selfdeveloped NWA for the measurement. Sensor measurement results of water - ethanol mixture are shown in Fig. 6. Measurements were made at a constant temperature of $30^{\circ} \mathrm{C}$ and demonstrate sufficient sensitivity to ethanol concentration.

\section{Conclusions}

Demonstrated technology for manufacturing of free-standing SU-8 structures provides a possibility to fabricate SU-8 based completely free-standing microfluidic sensor structures. Completed on SU-8 sensor electrodes demonstrate feasibility of standard lift-off metal layers processing on SU-8 coated wafers. Technology of "buried" in SU-8 metal electrodes provides a reliable structures with a considerably low cost processing. Control of SU-8 processing parameters makes it possible to achieve flexible and mechanically stable structures that can be applied for 3D attachment on a 3D molded interconnection device. Releasing steps completed with standard Omnicoat $^{\mathrm{TM}}$ processing gives an opportunity for considerably clean wet SU-8 releasing even with relatively large scale structures as it was demonstrated on Fig. 3 the whole 4" wafer was released without any structures damage. Sensor was assembled on 3D-MID platform integrating all electrical and fluidic interconnections. Fabricated sensor measurement results demonstrate high sensitivity to ethanol concentration analyzing water-ethanol mixtures.

\section{References}

[1] E. Barsoukov, J.R. Macdonald, Impedance Spectroscopy: Theory, Experiment, and Applications 27 January 2005, Pages 1-595, DOI: 10.1002/0471716243

[2] S. Gawad, L. Schild, Ph.Renaud, Micromachined impedance spectroscopy flow cytometer for cell analysis and particle sizing, Lab on a Chip Minituarization for Chemistry and Biology Volume 1, 76-82 (Issue 1 2001), DOI: 10.1039/b103933b

[3] R. Gómez, R. Bashir, A. Sarikaya, M.R. Ladisch, J. Sturgis, J.P. Robinson, T. Geng, A.K. Bhunia, H.L. Apple, S. Wereley, Microfluidic biochip for impedance spectroscopy of biological species, Biomedical Microdevices, 201-209 (Volume 3, Issue 3, 2001), DOI: 10.1023/A:1011403112850

[4] M.-P. Schmidt, A. Oseev, C. Engel, A. Brose, A. Aman, S. Hirsch, A Novel Design and Fabrication of Multichannel Microfluidic Impedance Spectroscopy Sensor for Intensive
Electromagnetic Environment Application, Proceeding of Eurosensors 2014, (2014), DOI: 10.1016/j.proeng.2014.11.272

[5] M.-P. Schmidt, A. Oseev, C. Engel, A. Brose, S. Hirsch, IMPEDANCE SPECTROSCOPY MICROFLUIDIC MULTICHANNEL SENSOR PLATFORM FOR LIQUID ANALYSIS, Proceeding of The 18th International Conference on Miniaturized Systems for Chemistry and Life Sciences, (2014), DOI: 10.13140/2.1.4254.8004

[6] C. Liu, Recent developments in polymer MEMS, Advanced Materials, 3783-3790 (Volume 19, Issue 22, 19. November 2007), DOI: 10.1002/adma.200701709

[7] B.-H.d Jo, L.M. Van Lerberghe, K.M. Motsegood, D.J. Beebe, Three-dimensional micro-channel fabrication in polydimethylsiloxane (PDMS) elastomer, Journal of Microelectromechanical Systems, 76-81 (Volume 9, Issue 1, March 2000), DOI: $10.1109 / 84.825780$

[8] S. Gawad, L. Schild, Ph. Renaud, Micromachined impedance spectroscopy flow cytometer for cell analysis and particle sizing, Lab on a Chip Minituarization for Chemistry and Biology, 76-82 (Volume 1, Issue 1, 2001), DOI: 10.1039/b103933b

[9] H. Lorenz, M. Despont, N. Fahrni, N. LaBianca, P. Renaud, P. Vettiger, SU-8: A low-cost negative resist for MEMS, Journal of Micromechanics and Microengineering, 121-124 (Volume 7, Issue 3, September 1997), DOI: 10.1088/0960$1317 / 7 / 3 / 010$

[10] H. Lorenz, M. Despont, N. Fahrni, J. Brugger, P. Vettiger, P. Renaud, High-aspect-ratio, ultrathick, negative-tone near-UV photoresist and its applications for MEMS, Sensors and Actuators, A: Physical, 33-39 (Volume 64, Issue 1, 1 January 1998), DOI: 10.1109/MEMSYS.1997.581916

[11] C.-H. Lin, G.-B. Lee, B.-W. Chang, G.-L. Chang, A new fabrication process for ultra-thick microfluidic microstructures utilizing SU-8 photoresist, Journal of Micromechanics and Microengineering, 590-597 (Volume 12, Issue 5, September 2002), DOI: 10.1088/0960$1317 / 12 / 5 / 312$

[12] J. Zhang, K.L. Tan, G.D. Hong, L.J. Yang, H.Q. Gong, Polymerization optimization of SU-8 photoresist and its applications in microfluidic systems and MEMS, Journal of Micromechanics and Microengineering, 20-26 (Volume 11, Issue 1, January 2001), DOI: 10.1088/0960$1317 / 11 / 1 / 304$

[13] R. Vilares, C. Hunter, I. Ugarte, I. Aranburu, J. Berganzo, J. Elizalde, L.J. Fernandez, Fabrication and testing of a SU-8 thermal flow sensor, Sensors and Actuators B, 411-417 (147 2010), DOI: 10.1016/j.snb.2010.03.054

[14] A. Ezkerra, L. J. Fernández, K. Mayora, J. M. Ruano-López, Fabrication of SU-8 free-standing structures embedded in microchannels for 
microfluidic control, J. Micromech. Microeng., 2264-2271 (17 2007), DOI: 10.1088/0960$1317 / 17 / 11 / 013$

[15] M. Agirregabiria, F. J. Blanco, J. Berganzo, M. T. Arroyo, A. Fullaondo, K. Mayora, J. M. RuanoLópez, Fabrication of SU-8 multilayer microstructures based on successive CMOS compatible adhesive bonding and releasing steps, Lab on a Chip - Miniaturisation for Chemistry and Biology, 545-552 (Volume 5, Issue 5, May 2005), DOI: 10.1039/b500519a

[16] P. Wang, K. Tanaka, S. Sugiyama, X. Dai, X. Zhao, Wet releasing and stripping SU-8 structures with a nanoscale sacrificial layer, Microelectronic Engineering, 2232-2235 (Volume 86, Issue 11, November 2009), DOI: 10.1016/j.mee.2009.03.079

[17] D.E. Pesántez, E.K. Amponsah, A.P. Gadre, Wet release of multipolymeric structures with a nanoscale release layer, Sensors and Actuators, B: Chemical, 426-430 (Volume 132, Issue 2, 16 June 2008), DOI: 10.1016/j.snb.2007.10.060 